\title{
Unprecedented Large Hyperpolarizability of Twisted Chromophores in Polar Media
}

\author{
Alexander J.-T. Lou, ${ }^{\S}$ Stefania Righetto, ${ }^{\dagger}$ Christopher Barger, ${ }^{\S}$ Cristiano Zuccaccia, ${ }^{*}, \ddagger \odot$

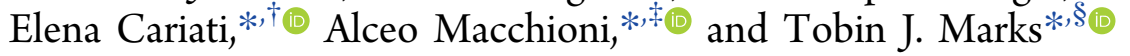 \\ ${ }^{\S}$ Department of Chemistry and the Materials Research Center, Northwestern University, 2145 Sheridan Road, Evanston, Illinois \\ 60208, United States \\ ${ }^{\dagger}$ Dipartimento di Chimica dell’Università di Milano and Unità di Ricerca dell’INSTM di Milano, Via Golgi 19, I-20133 Milano, Italy \\ ${ }^{\ddagger}$ Dipartimento di Chimica, Biologia e Biotecnologie and CIRCC, Università degli Studi di Perugia, Via Elce di Sotto, 8 I-06123 \\ Perugia, Italy
}

Supporting Information

ABSTRACT: Twisted intramolecular charge transfer (TICT) chromophores exhibit uniquely large second-order optical nonlinearities $(\mu \beta)$. However, their promise as electro-optic (E-O) materials is yet untapped, reflecting a strong tendency to aggregate in low-polarity media, leading to a dramatic fall in $\mu \beta$. Until now, TICT chromophores in deaggregating polar solvents suffered decreased response due to polarity-driven changes in electronic structure. Here we report a new series of benzimidazolium-based TICT chromophores with interaryl torsional angles in the range of 64-77 . The most twisted, B2TMC-2, exhibits a large $\mu \beta_{\mathrm{vec}}=$ $-26,000 \times 10^{-48}$ esu (at $1907 \mathrm{~nm}$ ) in dilute nonpolar $\mathrm{CH}_{2} \mathrm{Cl}_{2}$

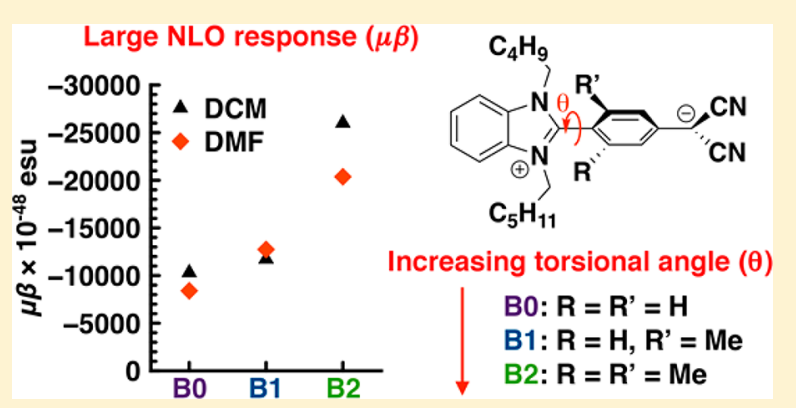
solution, which is maintained in polar DMF $\left(\mu \beta_{\text {vec }}=-20,370 \times 10^{-48}\right.$ esu $)$ as measured by DC electric field-induced second harmonic generation (EFISH). Sterically enforced interaryl torsional angles are confirmed by single-crystal X-ray diffraction and solution phase Nuclear Overhauser Effect (NOE) NMR, and spectroscopic characterization reveals a zwitterionic/aromatic ground state electronic structure associated with the high $\mu \beta$. We show that increasingly disrupted conjugation is correlated with increased $\mu \beta$ even at intermediate twist angles. The excellent performance and reduced aggregation in polar solvents opens new avenues for bridging microscopic and macroscopic chromophore performance.

\section{INTRODUCTION}

Second-order organic nonlinear optical (NLO) materials are of great interest for their ability to generate, process, and switch optical signals for applications such as image reconstruction and optical telecommunication. ${ }^{1,2}$ For useful optoelectronic devices to be created, the constituent organic chromophores must possess a large molecular hyperpolarizability $(\beta)$, optical transparency, chemical and thermal stability, and be effectively incorporated into device-ready materials. To date, organic materials have demonstrated excellent electro-optic coefficients in excess of $100 \mathrm{pm} / \mathrm{V}$ as compared to $32 \mathrm{pm} / \mathrm{V}$ in the ubiquitous inorganic device material $\mathrm{LiNbO}_{3}{ }^{1,3-7}$ Organic molecules have the attraction of being readily tuned structurally and electronically, and chromophores with large $\beta$ can be rationally designed using structure-property relationships developed over several decades.

In the past, the majority of second-order NLO designs have followed a simple paradigm in which the chromophores are composed of donor and acceptor functionalities bridged by a planar $\pi$-system. These "push-pull" systems typically possess a low-lying strongly polarized charge transfer (CT) state and can therefore be described by the simple "two-state model", which provides a qualitative relationship between $\beta$ and tunable molecular properties (eq 1$){ }^{8}$ Here, $\beta$ is related to the CT energy $\left(E_{\text {eg }}\right)$, transition dipole moment $\left(\mu_{\text {eg }}\right)$, and the change in state dipole moment $\left(\Delta \mu_{\mathrm{eg}}=\mu_{\mathrm{e}}-\mu_{\mathrm{g}}\right)$.

$$
\beta=\frac{\Delta \mu_{\mathrm{eg}} \mu_{\mathrm{eg}}{ }^{2}}{E_{\mathrm{eg}}{ }^{2}}
$$

Another approach, known as bond-length alternation (BLA), manipulates the contributions of CT limiting resonance forms to the ground state electronic structure to enhance $\beta .^{9-11} \mathrm{BLA}$ based chromophores (see CLD-1, Figure 1) generally contain donor and acceptor groups bridged by extended, planar, polyene backbones. Such structures have been critical to the elucidation of structure-property relationships and can exhibit $\mu \beta$ in excess of $17,600 \times 10^{-48}$ esu. $^{12}$ However, these BLA chromophores are often limited by photochemical and oxidative instability and rely on low-lying CT states, which erode the optical transparency window in the near-IR. ${ }^{12,13}$

Received: April 23, 2018

Published: June 18, 2018 


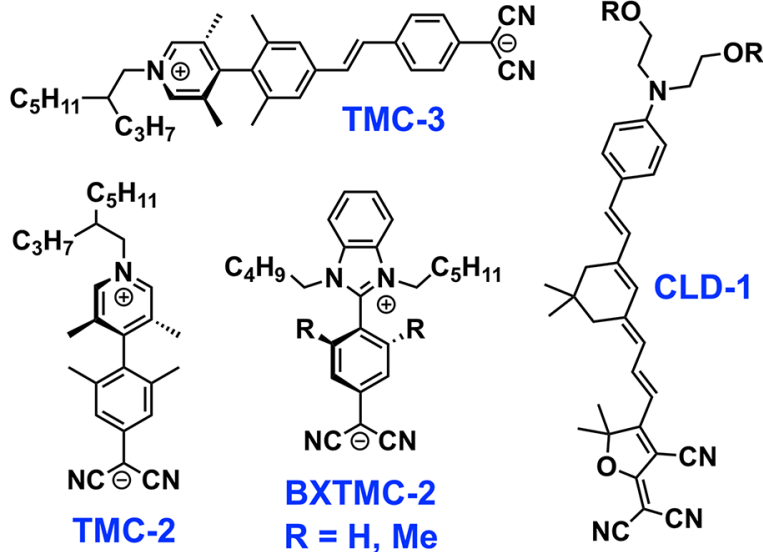

Figure 1. TICT chromophores TMC-2 and TMC-3, BLA-inspired CLD-1, and BXTMC-2 chromophores in this work.

Other approaches such as multidimensional chromophores, ${ }^{14,15}$ modulated conjugation, ${ }^{16}$ and auxiliary donor/ acceptors ${ }^{17}$ have also afforded promising increases in hyperpolarizability.

In 1997, Albert et al. challenged the notion that planarity was a prerequisite to large $\beta$, suggesting that a twisted intramolecular charge transfer (TICT) chromophore could surpass previous efforts. ${ }^{18}$ Following this report, TICT chromophore TMC-3 was experimentally shown to exhibit $\mu \beta_{\text {vec }}=-488,000 \times 10^{-48}$ esu (in dilute DCM solution) (Figure 2),,$^{5,18-22}$ exceeding the previous best $\mu \beta / \mathrm{MW}$ by a

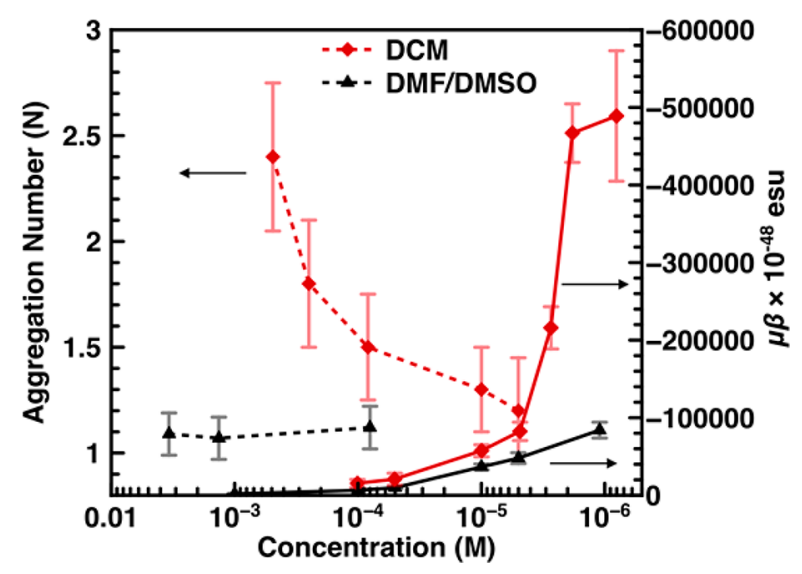

Figure 2. EFISH-derived $\mu \beta_{\text {vec }}$ (solid lines) and PGSE NMR-derived aggregation number (dotted lines) of TMC-3 as a function of concentration in DCM (red diamonds), DMSO- $d_{6}$ (black dotted line), and DMF (solid black lines). Figure reproduced from ref 5.

factor of $\sim 20 \times$ and approaching the fundamental limits on $\beta$ proposed by Kuzyk et al. ${ }^{1}$ The exceptional NLO response stems from a twisted biaryl bridge fragment and aromatic stabilization of the charge-separated ground state, which leads to a low-lying CT (small $E_{\mathrm{eg}}$ ) and large $\Delta \mu_{\mathrm{eg}}{ }^{23}$ Further experimental work on TICT chromophores focused on (1) the impact of torsional angle, ${ }^{22}$ (2) adding multiple twisted fragments, ${ }^{21}$ and (3) extending the conjugation. ${ }^{5}$

Nevertheless, although the NLO response of TICT chromophores is extraordinarily high in dilute, nonpolar solution, these chromophores suffer from dipole driven aggregation, yielding antiparallel dimers for which $\mu \beta=0 .{ }^{5}$ This tendency poses a challenge when attempting to translate solution-phase properties to host-guest polymer matrices, where high effective chromophore concentrations are required for useful bulk response. ${ }^{5,20}$ Although polar environments can provide stabilizing interactions that disrupt aggregate formation, they also severely depress the NLO response of the aforementioned TICT chromophores. For example, at low concentrations, the maximum $\mu \beta_{\text {vec }}$ of TMC-3 in polar DMF is approximately $1 / 5$ of that in DCM (Figure 2). ${ }^{20}$

The desire to translate molecular performance to bulk has been a long-standing goal within the community; past approaches include Langmuir-Blodgett films, poled polymers, layer-by-layer self-assembly, modification of the dielectric environment, and cochromophore incorporation. ${ }^{2,8,24-27}$ However, as we will discuss herein, structural modification of the chromophores themselves is, in this case, a necessary and productive strategy.

In the present study, we substitute the pyridinium acceptor fragment in TMC-2 with a benzimidazolium acceptor, leading to changes in electronic structure and allowing the strategic placement of two sterically demanding alkyl substituents. The steric interaction of these alkyl substituents with ortho-methyl groups creates a significant twist angle between the donor and acceptor $\pi$-ring planes. The new BXTMC-2 family (Scheme 1) exhibits excellent stability, solubility, and NLO performance. We show that, unlike previous TICT generations, these chromophores perform well in polar media, opening routes toward device incorporation that were previously unavailable for TICT chromophores. Furthermore, we characterize the key structural properties of these systems and use this information to augment previous observations of NLO response in relation to disrupted conjugation and intrinsic chromophore efficiency.

\section{RESULTS}

Having synthesized a series of benzimidazolium-based TICT chromophores with varied expected torsional angles, we investigate their solid-state and solution-phase properties. Particular attention is paid to changes accompanying increased steric encumbrance and to the relationship of BXTMC-2 to TMC-2. Single-crystal X-ray diffraction and NMR spectroscopy are used to determine the molecular geometry, including the biaryl torsional angle. Linear optical absorbance in a range of solvents, vibrational spectroscopy, and electrochemical measurements provide a detailed description of the electronic structure and the environmental dependence thereon. Nonlinear optical measurements using DC electric field-induced second harmonic generation (EFISH) are then used to determine $\mu \beta_{\mathrm{vec}}$ and to assess aggregation tendencies. Experimental details including synthetic and characterization procedures and relevant spectra are included in the Supporting Information (SI).

Synthesis of BXTMC-2 Chromophores. The synthesis of the BXTMC-2 family (Scheme 1) begins with condensation of phenylenediamine with the desired 4-bromo-benzaldehydes in the presence of air to form intermediates $\mathbf{1 A - 1 C}$. The acidic proton is then removed, and selective $\mathrm{N}$-alkylation is performed with 1-bromohexane. The aryl bromides in molecules $\mathbf{2 A}-\mathbf{2 C}$ can be efficiently converted to the corresponding dicyanomethanide functionalities via a $\mathrm{Pd}$ catalyzed coupling with malononitrile. The products of this reaction $(3 A-3 C)$ appear by NMR to be the zwitterionic isomer, where the acidic malononitrile proton migrates to the benzimidazole ring. As such, it is then necessary to treat structures $\mathbf{3 A}-\mathbf{3 C}$ with base prior to alkylation to yield the 
Scheme 1. Synthesis and Structures of BXTMC-2 Chromophores ${ }^{a}$<smiles>[R]c1cc(Br)cc([R])c1C=O</smiles>

$1 \mathrm{~A}-1 \mathrm{C}$<smiles>[R]c1cc(Br)cc([R])c1-c1nc2ccccc2n1CCCCCCCCCCC</smiles>

2A-2C (iii)

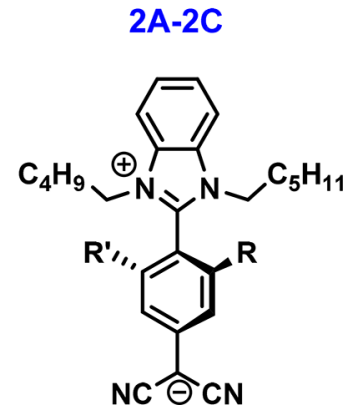

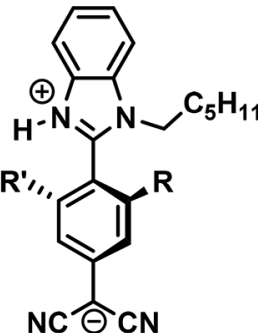

3A-3C

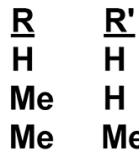

BOTMC-2

B1TMC-2 B2TMC-2

${ }^{a}$ Reagents and conditions: (i) phenylenediamine, 1,4-dioxane, bubbling air at $85{ }^{\circ} \mathrm{C}$; (ii) $\mathrm{KO}^{\mathrm{t}} \mathrm{Bu}, \mathrm{C}_{6} \mathrm{H}_{13} \mathrm{Br}, \mathrm{KI}$ (cat.), $\mathrm{THF}, 60{ }^{\circ} \mathrm{C}$; (iii) malononitrile, $\mathrm{NaO}{ }^{t} \mathrm{Bu}, \mathrm{Pd}\left(\mathrm{PPh}_{3}\right)_{4}, \mathrm{DME}, 85^{\circ} \mathrm{C}$; (iv) $\mathrm{KO}^{t} \mathrm{Bu}$ then $\mathrm{C}_{5} \mathrm{H}_{11} \mathrm{OTf}$ in acetone at $0{ }^{\circ} \mathrm{C}$.

final BXTMC-2 products and to perform alkylation at low temperature to ensure selectivity. The identity and purity of the final products was confirmed by a standard battery of spectroscopic and physical methods as described below and in the SI.

BXTMC-2 Solution Structures by ${ }^{1} \mathrm{H}$ and ${ }^{15} \mathrm{~N}$ NMR. ${ }^{1} \mathrm{H}$ NMR spectra of the BXTMC-2 chromophores in DMSO- $d_{6}$ (Figure 3) show two sets of signals in the aromatic region: (1)

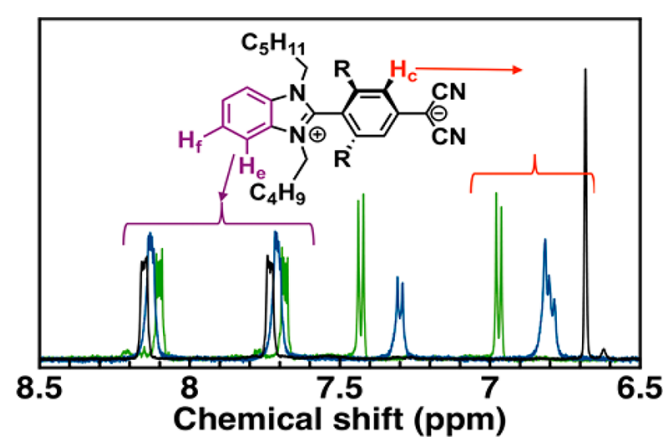

Figure 3. ${ }^{1} \mathrm{H}$ NMR spectra of BXTMC-2 in DMSO- $d_{6}$ : BOTMC-2 (green), B1TMC-2 (blue), and B2TMC-2 (black). Peaks related to $\mathrm{H}_{\mathrm{c}}$ are bracketed in red; $\mathrm{H}_{\mathrm{e}}$ and $\mathrm{H}_{\mathrm{f}}$ are in purple.

resonances at $\delta_{\mathrm{H}}=7.6-8.2 \mathrm{ppm}$ associated with the electrondeficient benzimidazolium acceptor fragment and (2) signals at $\delta_{\mathrm{H}}=6.6-7.5 \mathrm{ppm}$ for the electron-rich aryl donor fragment. $\mathrm{H}_{\mathrm{c}}$ in DMSO- $d_{6}$ shifts to higher field with increased biaryl torsion from B0TMC-2 $\left(\delta_{\mathrm{H}}=6.97 \mathrm{ppm}\right)$ to B1TMC-2 $\left(\delta_{\mathrm{H}} \sim\right.$ $6.82 \mathrm{ppm})$ and B2TMC-2 $\left(\delta_{\mathrm{H}}=6.68 \mathrm{ppm}\right)$, indicating an increased electron-richness in that molecular fragment. Both increasing the NOE NMR-determined twist angle (see more below) and addition of electron-donating methyl groups likely contribute to these shifts. The acceptor peaks $\left(\mathrm{H}_{\mathrm{e}}, \mathrm{H}_{\mathrm{f}}\right)$ do not appear to be very sensitive to such changes and exhibit $\Delta \delta_{\mathrm{H}}<$ $0.1 \mathrm{ppm}$ between B0- and B2TMC-2.

In contrast to the above ${ }^{1} \mathrm{H}$ aromatic chemical shift trends, the ${ }^{15} \mathrm{~N}$ chemical shifts $\left(\delta_{15 \mathrm{~N}}\right)$ of the benzimidazolium resonances (Figure 4, left) are displaced significantly and systematically within the BXTMC-2 series with $\delta_{15 \mathrm{~N}}$ increasing

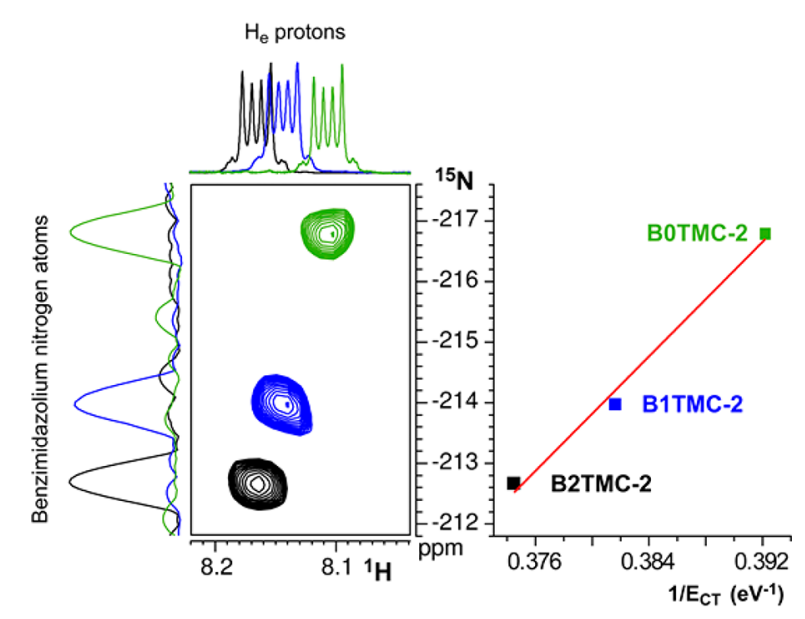

Figure 4. (left) Superimposed ${ }^{1} \mathrm{H},{ }^{15} \mathrm{~N}$ HMBC NMR spectra of BXTMC-2 in DMSO- $d_{6}$. (right) Trend of $\delta_{15 \mathrm{~N}}(\mathrm{ppm})$ as a function of the inverse of the optically determined HOMO-LUMO energy gap $\left(1 / E_{\mathrm{CT}}\right)$.

from B0- (-216.8 ppm) to B1- (-214.0 ppm) and B2TMC-2 $(-212.7 \mathrm{ppm})$. The observed $\delta_{15 \mathrm{~N}}$ trend is determined by the paramagnetic contribution as is usual for nuclei heavier than ${ }^{1} \mathrm{H}$. Consistently, a good linear correlation is obtained when plotting $\delta_{15 \mathrm{~N}}$ versus the inverse of the optically determined HOMO-LUMO energy gap $\left(E_{\mathrm{CT}}\right.$, Table 2$)$ according to the Ramsey equation (Figure 4, right). ${ }^{28-30}$ This $\delta_{15 \mathrm{~N}}$ trend also reasonably reflects increasing accumulation of positive charge at the benzimidazolium nitrogen atoms on progressing from BOTMC-2 to B2TMC-2 ${ }^{31-33}$ and indicates an increasing contribution of the aromatic resonance form to the groundstate structure. ${ }^{34,35}$ Comparison of relative $\delta_{15 \mathrm{~N}}$ values indicates a larger variation of the biaryl torsion between B0- 
and B1TMC-2 than between B1- and B2TMC-2 in agreement with NOE data (see below).

Crystallographic Characterization of BXTMC-2 Chromophores. Single-crystal diffraction characterization provides insight both into the molecular geometries of the chromophores as well as the modes of solid-state intermolecular interactions operative (Figures 5 and 6). Large biaryl torsional

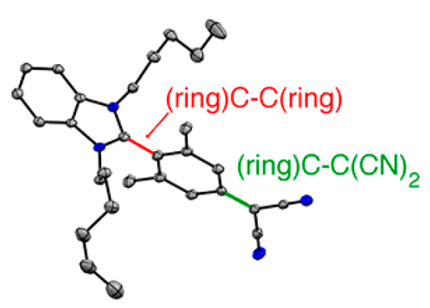

Figure 5. ORTEP drawing of chromophore B2TMC-2 with 30\% probability ellipsoids showing key bond metrical parameters. Note that the orientation of the structure causes partial overlap of two alkyl group carbon atoms.

\section{BOTMC-2}
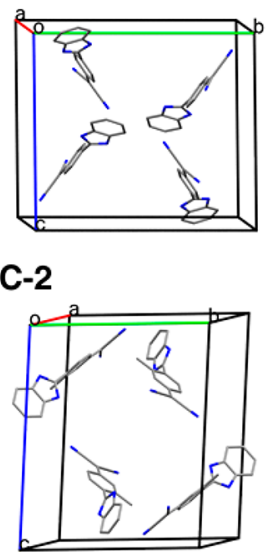

B2TMC-2

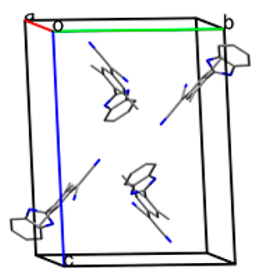

B1TMC-2
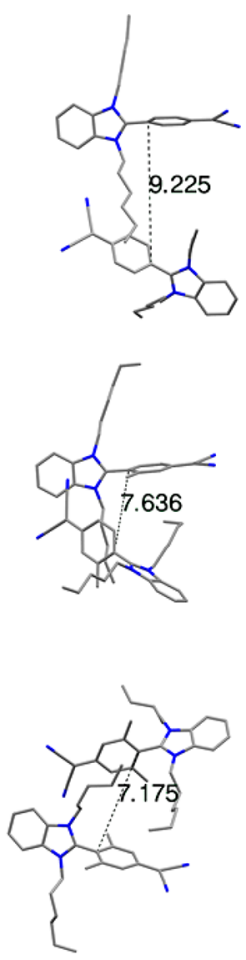

Figure 6. Crystal structures of the BXTMC-2 chromophore series. (left) Packing of the tetrameric centrosymmetric unit cells (alkyl groups excluded for clarity). (right) Closest-packed dimeric structures with relevant packing distances measured in $\AA$.

angles of $70^{\circ}, 89^{\circ}$, and $79^{\circ}$ are observed for B0-, B1-, and B2TMC-2, respectively, confirming the steric contribution of both $\mathrm{H} / \mathrm{CH}_{2}$ and $\mathrm{CH}_{3} / \mathrm{CH}_{2}$ repulsions. Two key bond distances are also examined (Figure 5, Table 1). First, the bridging (ring)C-C(ring) bond distances are 1.465(4), 1.474(8), and 1.478(4) $\AA$ for B0-, B1-, and B2TMC-2, respectively. These bond lengths are more similar to those observed for bimesitylene $(1.501 \AA)^{36}$ and biaryls $(1.487 \AA)^{37}$ than typical $\mathrm{C}=\mathrm{C}$ bonds $(\sim 1.33 \AA){ }^{38}$ indicating that these molecules are best described by linked aromatic structures rather than as quinones. The comparison of B0- and B2TMC-
Table 1. Crystallographic Bond and Intermolecular Packing Distances

\begin{tabular}{lccc} 
& \multicolumn{3}{c}{ distance $(\AA)$} \\
\cline { 2 - 4 } & (ring)C-C(ring) & $($ ring $) \mathrm{C}-\mathrm{C}(\mathrm{CN})_{2}$ & packing distance \\
B0TMC-2 & $1.465(4)$ & $1.445(4)$ & $9.225(6)$ \\
B1TMC-2 & $1.474(8)$ & $1.438(8)$ & $7.636(8)$ \\
B2TMC-2 & $1.478(4)$ & $1.450(4)$ & $7.175(4)$ \\
\hline
\end{tabular}

2 structures indicates that the increased torsion arising from added methyl groups leads to a slight elongation of the (ring)C-C(ring) bond likely due to a decrease in its double bond character. The uncertainty in B1TMC-2 bond distances precludes comparison with the other structures.

The second key bond distances, (ring) $\mathrm{C}-\mathrm{C}(\mathrm{CN})_{2}$, are found to be 1.445(4), 1.438(8), and 1.450(4) $\AA$ for B0-, B1-, and B2TMC-2, respectively (Table 1 ). These values are longer than the analogous bond lengths found in quinoidal TCNQ $(1.373 \AA),{ }^{39}$ suggesting that the dicyanomethanide group supports significant negative charge. The increase in bond length between B1- and B2TMC-2 indicates a decrease in double-bond nature of the $\operatorname{ring}(\mathrm{C})-\mathrm{C}(\mathrm{CN})_{2}$ bond consistent with increased aromatic character of the structure (see Figure 11). The similarity of B0- and B1TMC-2 $\operatorname{ring}(\mathrm{C})-\mathrm{C}(\mathrm{CN})_{2}$ distances may be a coincidental result of different packing structures; BOTMC-2 exhibits close contact between donor and acceptor of adjacent molecules, potentially stabilizing the negative charge.

The BXTMC-2 crystal structures all exhibit antiparallel dimer packing within the centrosymmetric tetramers that constitute the unit cell (Figure 6). The distance between the bridging carbon on the donor ring of the antiparallel aligned pairs provides an estimate of the closeness of the intermolecular interaction. This distance contracts on progressing from B0TMC-2 (9.220(6) ^) to B1TMC-2 (7.636(8) $\AA$ ) and B2TMC-2 (7.175(4) Å), suggesting stronger intermolecular interactions, particularly in B2TMC2. This trend is in agreement with a computed increase in ground-state dipole moment, which is likely a driving force in these interactions. There is no evidence of cocrystallized solvent associated with these structures, and the unit cells lack solvent-accessible voids.

Nuclear Overhauser Effect (NOE) NMR Characterization of Chromophore Structures in Solution. ${ }^{1} \mathrm{H}-{ }^{1} \mathrm{H}$ NOE NMR experiments, which measure through space dipolar coupling, were undertaken in DMSO- $d_{6}$ to estimate the average twist angle in solution for the BXTMC-2 chromophore series. For B2TMC-2, NOE measurements were carried out by irradiating the $\mathrm{Me}$ singlet $\left(\delta_{\mathrm{H}}=1.93 \mathrm{ppm}\right.$ at $\left.298 \mathrm{~K}\right)$ and measuring NOEs at both $\mathrm{H}_{\mathrm{a}}$ and $\mathrm{H}_{\mathrm{c}}$ (Scheme 2). For BOTMC2 , the $\mathrm{H}_{\mathrm{d}}$ doublet $\left(\delta_{\mathrm{H}}=7.44 \mathrm{ppm}\right.$ at $\left.298 \mathrm{~K}\right)$ was irradiated, and NOEs were measured on $\mathrm{H}_{\mathrm{a}}$. In the case of B1TMC-2, both the Me singlet $\left(\delta_{\mathrm{H}}=2.02 \mathrm{ppm}\right.$ at $\left.298 \mathrm{~K}\right)$ and the $\mathrm{H}_{\mathrm{d}}$ doublet $\left(\delta_{\mathrm{H}}=7.31 \mathrm{ppm}\right.$ at $\left.298 \mathrm{~K}\right)$ were irradiated, and the following NOEs were quantified: $M e-H_{c}, M e-H_{a}$ and $\mathrm{H}_{d}-\mathrm{H}_{b}$. From the measured NOEs, average values of cross relaxation rate constants $\left(\left\langle\sigma_{\mathrm{IS}}\right\rangle\right)^{40}$ at different temperatures (Figure S1) and average internuclear distances $\left\langle\mathrm{r}_{\mathrm{IS}}\right\rangle^{41}$ were obtained (Table S4) using the methodology described previously. ${ }^{5}$

For the average internuclear distance measured in solution between aromatic (Me or $\mathrm{H}_{\mathrm{d}}$ groups) and benzimidazole $\left(\mathrm{H}_{\mathrm{a}}\right.$ and $\mathrm{H}_{\mathrm{b}}$ protons) moieties to be correlated with the average twist angle $(\theta)$ in solution, average internuclear distances were 
Scheme 2. BXTMC-2 Chromophore Structures and Labeling Scheme Showing syn and anti Configurations

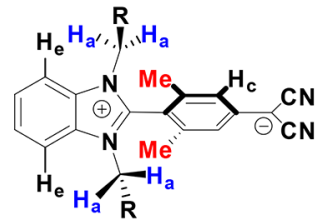

B2TMC-2 anti

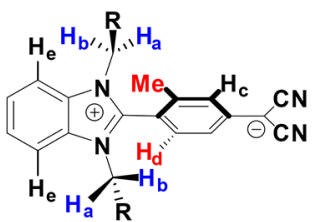

B1TMC-2 anti

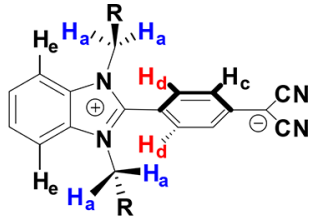

BOTMC-2 anti

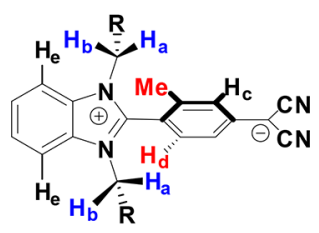

B1TMC-2 syn computed under $\mathrm{r}_{\mathrm{IS}}{ }^{-6}$ averaging ${ }^{42}$ for seven static conformations $\left(\theta\right.$ approximately equal to $60^{\circ}, 65^{\circ}, 70^{\circ}, 75^{\circ}, 80^{\circ}, 85^{\circ}$ and $90^{\circ}$ ) starting from the solid-state X-ray structures of the BXTMC-2 series. Considering the simplified approach detailed in the Supporting Information, the computed average distances, reported in Figure 7, are somewhat biased toward

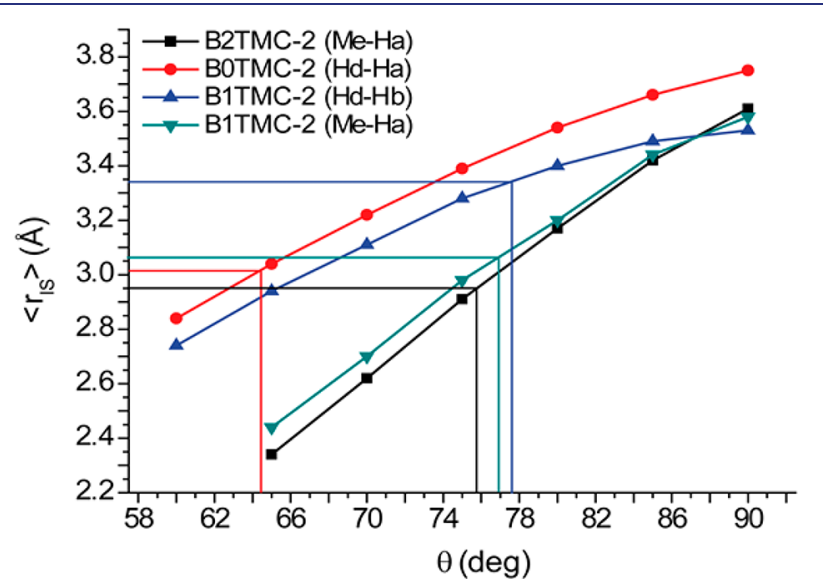

Figure 7. Computed average distance $\left\langle\mathrm{r}_{\mathrm{IS}}\right\rangle$ for BOTMC-2 $\left(\mathrm{H}_{\mathrm{d}}-\mathrm{H}_{\mathrm{a}}\right)$, B1TMC-2 (Me- $\mathrm{H}_{\mathrm{a}}$ and $\left.\mathrm{H}_{\mathrm{d}}-\mathrm{H}_{\mathrm{b}}\right)$, and B2TMC-2 $\left(\mathrm{Me}-\mathrm{H}_{\mathrm{a}}\right)$ chromophores in the solid state as a function of dihedral twist angle $(\theta)$. The cross points indicate the experimentally NOE-derived distances and the corresponding average twist angle in solution.

shorter distances (i.e., toward the distance of closest approach); consequently, the derived twist angles in solution (cross points in Figure 7) are somewhat underestimated and should be better considered as a lower limit for $\theta$. There is no doubt that BOTMC-2 exhibits the smallest twist angle $\left(64^{\circ}\right)$ in solution (Figure 7). However, B1- and B2TMC-2 are found to have very similar twist angles $\left(77-78^{\circ}\right.$ and $76^{\circ}$, respectively) according to the present analysis. From this analysis, it is clear that both $\mathrm{Me} / \mathrm{CH}_{2}$ and $\mathrm{H} / \mathrm{CH}_{2}$ interactions play a significant role in biaryl torsion. The similarity of the B1- and B2TMC-2 torsional angles is addressed in the Discussion.

Infrared (FTIR) Vibrational Spectroscopy. BXTMC-2 compounds were characterized by FTIR both as solids and in solution. The key feature of these spectra is the characteristic $\mathrm{C} \equiv \mathrm{N}$ stretching, $\quad v(\mathrm{C} \equiv \mathrm{N})$, with a lower energy side component, presumably the symmetrically and antisymmetri- cally coupled modes (Figure 8$)$. In the solid state, B1- and B2TMC-2 exhibit $v(\mathrm{C} \equiv \mathrm{N})=\left(2168,2133 \mathrm{~cm}^{-1}\right)$, whereas
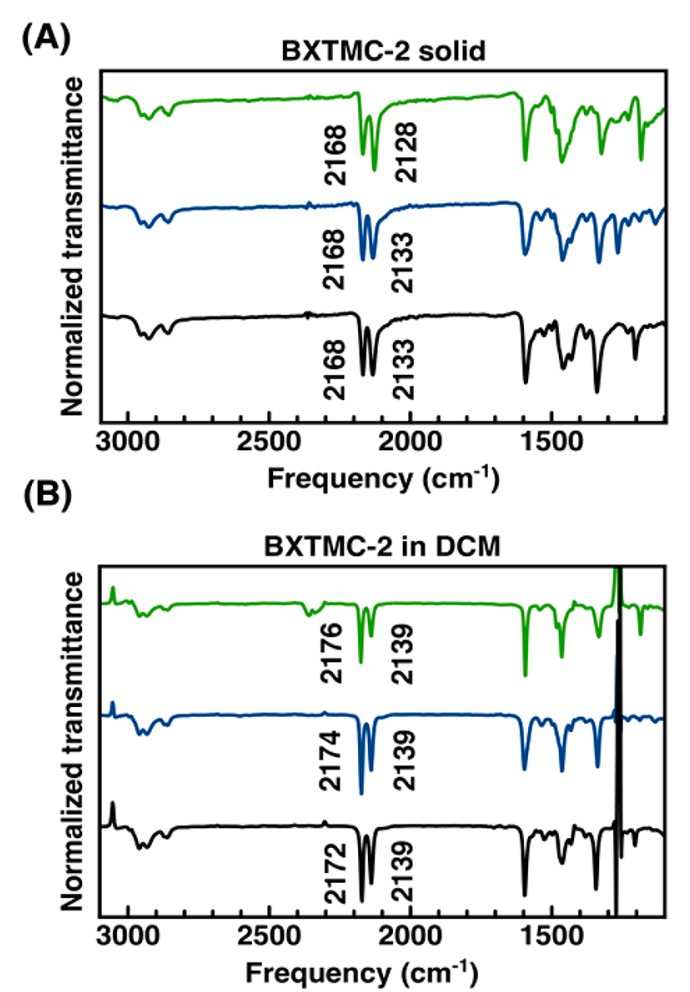

Figure 8. FTIR vibrational spectra of B0TMC-2 (green), B1TMC-2 (blue), and B2TMC-2 (black): (A) solid (ATR), (B) DCM solution.

B0TMC-2 exhibits a slightly lower energy sideband at 2128 $\mathrm{cm}^{-1}$. The energy and splitting of the bands is similar to a phenyl malononitrile anion $\left(v(\mathrm{C} \equiv \mathrm{N})=2163,2117 \mathrm{~cm}^{-1}\right){ }^{43}$ indicating that the dicyanomethanide groups in BXTMC-2 bear a large amount of electron density.

In DCM solution, all BXTMC-2 chromophore molecules display significantly higher energy $v(\mathrm{C} \equiv \mathrm{N})$ stretching modes than as solids, and $v(\mathrm{C} \equiv \mathrm{N})$ falls in energy from B0- $(2176$ $\left.\mathrm{cm}^{-1}\right)$ to B1- $\left(2174 \mathrm{~cm}^{-1}\right)$ and B2TMC-2 $\left(2172 \mathrm{~cm}^{-1}\right)$. This trend indicates a slight increase in electron density on the dicyanomethanide group accompanying the addition of methyl groups. Similar results were obtained in DMF with the $v(\mathrm{C} \equiv$ $\mathrm{N})$ band increasing from $2170 \mathrm{~cm}^{-1}$ in B2TMC-2 to 2172 $\mathrm{cm}^{-1}$ in BOTMC-2 (Figure S10). The sideband is higher in energy in solution than in the solid state $\left(2139 \mathrm{~cm}^{-1}\right.$ in DCM, $2135-2137 \mathrm{~cm}^{-1}$ in DMF). These observations suggest that the BXTMC-2 chromophores exhibit predominantly zwitterionic character, which increases in the order DCM $<$ DMF $<$ solid.

Linear Optical Absorption Spectroscopy as a Function of Solvent. The solution optical absorption of the BXTMC-2 family (Figure 9A) all exhibit a broad low-lying charge transfer (CT) absorption (434-408 nm) and a higher energy transition $(315-328 \mathrm{~nm})$. The intensity of the CT peak decreases from $\varepsilon=24,928 \mathrm{M}^{-1} \mathrm{~cm}^{-1}$ in BOTMC-2 to $\varepsilon=8156$ $\mathrm{M}^{-1} \mathrm{~cm}^{-1}$ in B2TMC-2. Such a reduction in transition intensity suggests increasingly disrupted conjugation, which is in agreement with reduced NOE and crystallographic torsional angles for B0TMC-2 but not for B1 and B2TMC-2. This discrepancy is addressed in detail in the Discussion. By integrating the CT peaks (see Supporting Information), one 
(A)

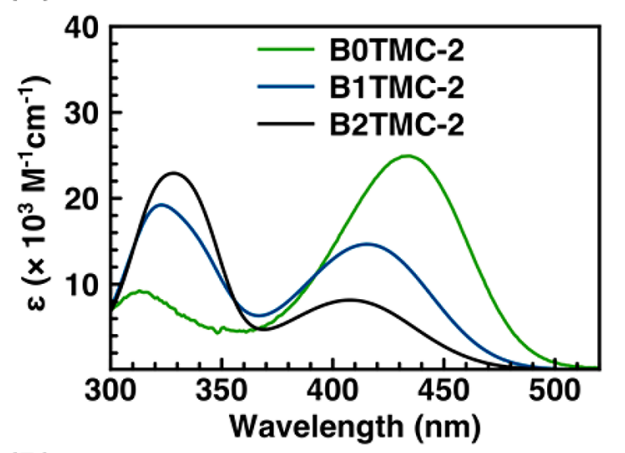

(B)

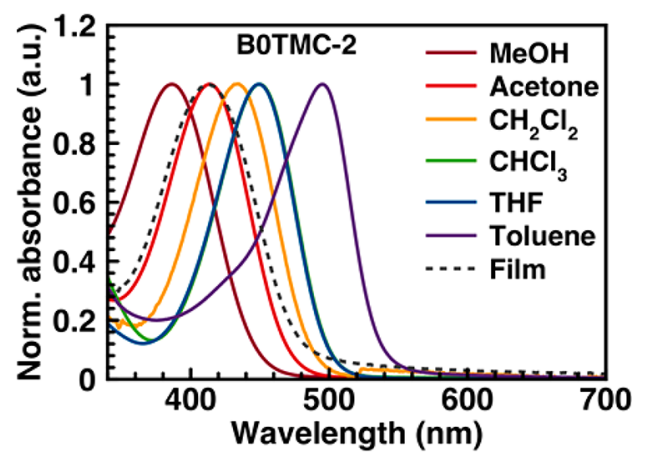

Figure 9. UV-vis linear optical absorption spectra of BXTMC-2 chromophores: (A) BXTMC-2 chromophores in DCM solution; (B) BOTMC-2 in the indicated solvents and as film.

can also extract transition moments $\left(\mu_{\mathrm{eg}}\right)$, which reveal relatively large transition strengths of $5.45 \mathrm{D}$ in B0TMC-2 and $3.43 \mathrm{D}$ in B2TMC-2 as compared to $2.1 \mathrm{D}$ in TMC-2 (Table 2).

The fall in transition intensity is accompanied by an $\sim 27 \mathrm{~nm}$ hypsochromic shift from B0TMC-2 to B1TMC-2 to B2TMC2 in DCM. The higher energy excitation, attributable to a subfragment transition, also undergoes an $\sim 13 \mathrm{~nm}$ bathochromic shift along with a slight increase in transition strength from B0TMC-2 to B2TMC-2. The behavior in Figure 9A is typical of TICT chromophores: as conjugation is disrupted, both subfragments of the molecule become isolated, and their own characteristic transitions begin to dominate. ${ }^{22}$

Solvent-dependent spectra (Figure 9B) reveal negative solvatochromic shifts of the CT band, meaning that CT wavelength $\left(\lambda_{\mathrm{CT}}\right)$ increases with solvent polarity (the shift between $\mathrm{MeOH}$ and $\mathrm{CHCl}_{3}$ is reported as $\Delta \lambda$ in Table 2). Such shifts indicate that the ground-state dipole moment is larger than that of the first excited state $\left(\Delta \mu_{\mathrm{eg}}<0\right)$ and therefore is stabilized by the increase in solvent polarity. The value of $\lambda_{\mathrm{CT}}$ is determined by Gaussian fitting of the CT peak, which eliminates the effect of the subfragment transition on the CT peak position (Table 2). The McRae equation then provides a simple means to calculate $\Delta \mu_{\text {eg }}$ by plotting the change in $E_{\mathrm{CT}}$ in various solvents (Figure S4) against a solvent polarity function $f(\varepsilon, n)$. ${ }^{44-46}$

$$
v-v_{0}=-m \times f\left(e, n^{2}\right)
$$

where $n$ is the refractive index, $\varepsilon$ is the solvent dielectric constant, and $v-v_{0}$ is the difference between the CT frequency (in $\mathrm{cm}^{-1}$ ) in a given solvent and vacuum. By relating the slope $m$ to $\Delta \mu_{\mathrm{eg}}$, an increase in $\Delta \mu_{\mathrm{eg}}$ from $-12.3 \mathrm{D}$ in B0TMC-2 to $-13.7 \mathrm{D}$ in B2TMC-2 is calculated (Table 2). More details of this analysis can be found in the SI. UV-vis absorption spectra of neat films (Figure S3) are found in all cases to be similar to those in acetone solution, demonstrating that the molecules behave comparably as solids and in relatively polar solutions.

BXTMC-2 Cyclic Voltammetry. Chromophores B1- and B2TMC-2 exhibit reversible reduction at -2.03 and $-2.10 \mathrm{~V}$, respectively, and the reduction of BOTMC-2 is irreversible at $-1.94 \mathrm{~V}$ (Table 2). Oxidation is irreversible for all chromophores with an onset between 0.24 and $0.36 \mathrm{~V}$. From the oxidation and reduction potentials, the HOMO-LUMO gaps are estimated to be 2.18, 2.39, and $2.44 \mathrm{eV}$ for B0-, B1-, and B2TMC-2, respectively, on average $\sim 0.3 \mathrm{eV}$ lower than the optical $E_{\mathrm{CT}}$, possibly reflecting some mixed character in the optical transitions and solvation reorganization in redox processes. $^{47}$

NLO Measurements. Measurements of $\mu \beta_{\text {vec }}$ the product of the chromophore dipole moment $(\mu)$, and the vector part of the molecular first-order hyperpolarizability $\beta_{\text {vec }}$ tensor along the $\mu$ direction were performed by the solution-phase DC EFISH method, which provides direct information on the intrinsic molecular nonlinear optical (NLO) response via eq 3.

$$
\gamma_{\mathrm{EFISH}}=(\mu \beta / 5 k T)+\gamma(-2 \omega ; \omega, \omega, 0)
$$

where $\mu \beta / 5 k T$ is the dipolar orientational contribution, and $\gamma(-2 \omega ; \omega, \omega, 0)$, the third-order term at frequency $\omega$ of the incident light, is the electronic contribution to $\gamma_{\mathrm{EFISH}}$, which is negligible for molecules of the type investigated here. ${ }^{48}$ EFISH measurements at $1907 \mathrm{~nm}$ were performed over a concentration range in DCM and DMF to determine (1) trends in the monomeric response of the BXTMC-2 chromophores in highly dilute solutions, (2) the dependence on solvent environment, and (3) the effect of aggregation in different solvent environments. It is well-known that highly polar TICT chromophores have a strong tendency to aggregate in solution, ${ }^{5}$ so the most dilute reliable measurement for each chromophore was taken to be representative of monomeric

\begin{tabular}{|c|c|c|c|c|c|c|c|c|}
\hline & \multirow[b]{2}{*}{$\lambda_{\mathrm{CT}}(\mathrm{nm})$} & \multirow[b]{2}{*}{$E_{\mathrm{CT}}\left(\mathrm{DCM}^{c}\right)(\mathrm{eV})$} & \multirow[b]{2}{*}{$\Delta \lambda(\mathrm{nm})^{d}$} & \multicolumn{2}{|c|}{$E(\mathrm{ACN})^{b}$} & \multirow[b]{2}{*}{$\mu_{\mathrm{eg}}(\mathrm{D})^{e}$} & \multirow[b]{2}{*}{$\mu_{\mathrm{g}}(\mathrm{D})^{f}$} & \multirow[b]{2}{*}{$\Delta \mu_{\mathrm{eg}}(\mathrm{D})^{g}$} \\
\hline & & & & $E_{\text {ox }}(\mathrm{V})$ & $E_{\text {red }}(\mathrm{V})$ & & & \\
\hline ВоTMC-2 & 434 & 2.55 & -64 & 0.24 & -1.94 & 5.45 & 21.2 & -12.3 \\
\hline B1TMC-2 & 415 & 2.62 & -65 & 0.36 & -2.03 & 4.72 & 23.5 & -13.0 \\
\hline В2ТМC-2 & 408 & 2.67 & -57 & 0.34 & -2.10 & 3.43 & 24.6 & -13.7 \\
\hline TMC- $^{h}$ & 556 & 2.18 & $\sim-180$ & 0.39 & -1.56 & 2.10 & 29.8 & -21.4 \\
\hline
\end{tabular}

Table 2. BXTMC-2 Optical Absorption and Electrochemical Data ${ }^{a}$

${ }^{a}$ All values reported are measured in DCM solution except where noted otherwise. ${ }^{b}$ Measured from signal onset. ${ }^{c}$ Optical HOMO-LUMO gap estimated from onset of $\mathrm{CT}$ absorption. ${ }^{d}$ Solvent shift from $\mathrm{CHCl}_{3}$ to $\mathrm{MeOH}$. ${ }^{e}$ Extracted from linear absorption, see SI for details. ${ }^{f} \mathrm{Calculated}$ using CAM-B3LYP/6-31G**. ${ }^{g}$ Extracted from linear absorption using the McRae equation, see SI for details. ${ }^{h}$ Data from ref 5. 
$\mu \beta_{\text {vec }}$. Note that this value reflects the lower bound of $\mu \beta_{\text {vec }}$ particularly in the case of B1TMC-2 for which larger values were recorded at higher dilution but with greater uncertainty. Measurements in DCM reveal very large monomeric $\mu \beta_{\text {vec }}=$ $-26,000 \times 10^{-48}$ esu for B2TMC-2 and $\mu \beta_{\text {vec }}$ of $-11,730$ and $-10,300 \times 10^{-48}$ esu for B1TMC-2 and B0TMC-2, respectively (Figure 10A). The response of B2TMC-2 is on the order of the monomeric response of TMC-2 (Figure 1; $\left.\mu \beta_{\text {vec }}=-24,000 \times 10^{-48} \mathrm{esu}\right)$ despite increases in $E_{\mathrm{CT}}$ and a reduced $\Delta \mu_{\mathrm{eg}}$.

(A)

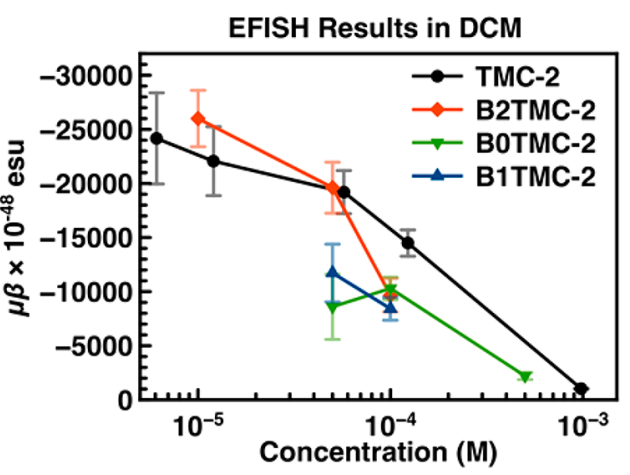

(B)

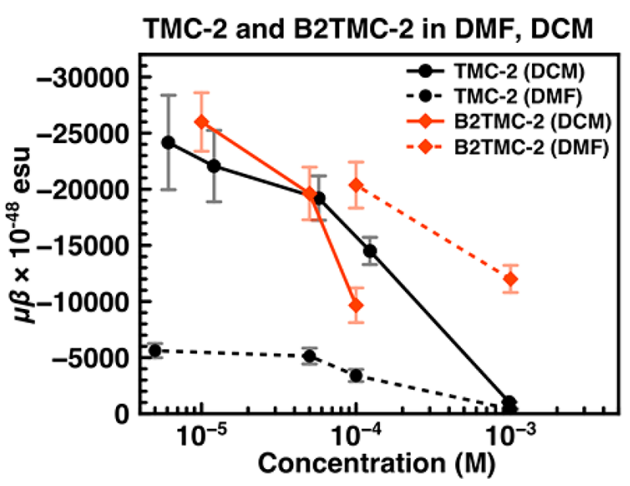

Figure 10. EFISH measurements of BXTMC-2 chromophores at $1907 \mathrm{~nm}$ (A) in DCM with TMC-2 data from ref 5; (B) comparison of $\mu \beta$ in DCM (solid lines) and DMF (dashed lines) for B2TMC-2 and TMC-2.

EFISH measurements on B2TMC-2 solutions in polar DMF (Figure 10B) reveal an NLO response of $\mu \beta_{\text {vec }}=-20,370 \times$ $10^{-48}$ esu, which is similar to the measured value in DCM and $\sim 4 \times$ higher than TMC-2 $\left(-5,620 \times 10^{-48}\right.$ esu) under the same conditions. B1TMC-2 shows a similarly high value of $\mu \beta_{\text {vec }}=-12,740 \times 10^{-48}$ esu in DMF compared to $-11,730 \times$ $10^{-48}$ esu in DCM (Table 3). These observations are in sharp contrast to all previous TICT chromophores, which suffer massively decreased NLO responses in polar solvents (Figures 1 and $10 \mathrm{~B})$.

In DCM solutions, a significant decrease in $\mu \beta_{\text {vec }}$ is observed with increasing concentration in the range of $10^{-5}-10^{-4} \mathrm{M}$ for B1- and B2TMC-2, and $10^{-4}-10^{-3} \mathrm{M}$ for BOTMC-2. This behavior is consistent with the formation of centrosymmetric aggregates (for which $\mu \beta=0$ ) and mirrors previous observations on TMC-2 in DCM (Figure 10). The concentration dependence of NLO response is more pronounced in the most twisted chromophore (B2TMC-2) than in the least twisted (BOTMC-2), likely reflecting the dipole moment enhancement that accompanies biaryl torsion.
Table 3. EFISH Results for BXTMC-2 Chromophores in DCM and DMF at $1907 \mathrm{~nm}$

\begin{tabular}{lcccccc} 
& \multicolumn{2}{c}{$\mu \beta\left(\mathrm{esu} \times 10^{-48}\right)^{a}$} & & \multicolumn{2}{c}{$\beta\left(\mathrm{esu} \times 10^{-30}\right)^{b}$} \\
\cline { 2 - 3 } \cline { 5 - 6 } & DCM & DMF & & DCM & DMF \\
B0TMC-2 & $-10,300$ & $-8,400$ & & -486 & -396 \\
B1TMC-2 & $-11,730$ & $-12,740$ & & -499 & -542 \\
B2TMC-2 & $-26,000$ & $-20,370$ & & $-1,056$ & -828 \\
TMC-2 $^{c}$ & $-24,000$ & $-5,620$ & & -805 & -189
\end{tabular}

${ }^{a}$ Highest dilution with reliable data is used to estimate $\mu \beta_{\text {vec }}$. ${ }^{b}$ Calculated using DFT-derived dipole moments in Table $1 .{ }^{c}$ Data from ref 5 .

The use of more polar DMF appears to mitigate these effects and shifts the decreases in NLO response toward higher concentrations (Figure 10B).

\section{DISCUSSION}

Synthesis of the BXTMC-2 Family. The synthetic route in Scheme 1 used to create BXTMC-2 chromophores offers some significant advantages over the previous generations: (1) the final product here can be obtained in only 4 steps, (2) there is an opportunity to introduce asymmetric or functionalized side chains, and (3) introduction of the steric bulk in two separate steps leads to high yields and a further degree of synthetic flexibility. By forming the benzimidazolium acceptor group via annulation rather than coupling, we avoid repeated use of aryl halide functionality, significantly reducing the number of required synthetic steps. Furthermore, the BXTMC-2 family exhibits excellent thermal stability with thermogravimetric analysis-derived decomposition temperatures in excess of $310{ }^{\circ} \mathrm{C}$ (Figure S5).

BXTMC-2 Electronic Structure. The ground-state electronic structure of BXTMC-2 chromophores can be represented as a mixture of zwitterionic/aromatic (ZA) and quinoidal/neutral (NQ) resonance forms (Figure 11). As

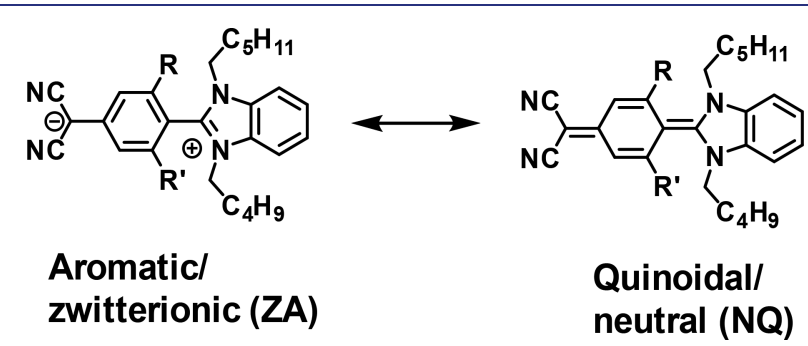

Figure 11. Quinoidal and aromatic contributions to the BXTMC-2 electronic structure.

shown in the Results, all three BXTMC-2 chromophores are best described by the ZA structure. The crystallographic (ring) $\mathrm{C}-\mathrm{C}$ (ring) bond lengths are similar to those found in biaryls and bimesitylene, and vibrational frequencies of the $\mathrm{C}(\mathrm{CN})_{2}$ group show that the donor group bears an essentially full negative charge. This is consistent with previous work by Isborn et al., which notes that even at modest twist angles, aromatic stabilization of the donor and acceptor imparts dominant ZA character in similar molecules. ${ }^{49}$ However, there is also evidence here supporting a subtle increase in $\mathrm{ZA}$ character from B0- to B1- and B2TMC-2. Shifts of the ${ }^{1} \mathrm{H}$ and ${ }^{15} \mathrm{~N}$ NMR resonance positions, increased solvatochromic shifts, lengthening of crystallographically derived (ring) $\mathrm{C}-\mathrm{C}$ (ring) bond distances, and increased $v(\mathrm{C} \equiv \mathrm{N})$ energies support the 
trend in ZA character increasing in the progression B0- $<$ B1< B2TMC-2.

Although both crystallographic and NOE characterization data definitively show that $\mathrm{Me} / \mathrm{CH}_{2}$ interactions lead to larger twist angles $(\theta)$ than do $\mathrm{H} / \mathrm{CH}_{2}$, they also indicate similar $\theta$ for B1- and B2TMC-2. This is in contrast to the decrease in the measured CT transition moment $\left(\mu_{\mathrm{eg}}\right)$ for $\mathbf{B} 2$ - versus B1TMC-2, which suggests a reduction of conjugation. The reason for this observed discrepancy may relate to the dynamic nature of $\theta$. Although the two chromophores have similar average $\theta$ by NOE, they may be able to access a range of $\theta$ through thermal excitation of the vibrational ring-twisting mode. As added $\mathrm{Me} / \mathrm{CH}_{2}$ interactions are shown to increase twisting, it is reasonable that B2TMC-2, with twice the steric resistance, is less able to access smaller $\theta$ values than B1TMC$\mathbf{2}$ at a given temperature. Thus, despite having similar average $\theta$, thermal energy may more easily populate less twisted configurations with large $\mu_{\text {eg }}$ for B1TMC-2, leading to larger observed CT absorption than for B2TMC-2. It should be noted that quantum computation indicates that the BXTMC-2 chromophores will exhibit similar $\theta$ values in DCM and DMSO (Tables S9 and S10), and so we have successfully accessed intermediate $\theta$ in polar and nonpolar solution, which are less than $80^{\circ}$, but still deviate significantly from planarity.

Comparison to TMC-2 and Previous TICT Chromophores. Comparison to other TICT chromophores sheds light on the impact of the benzimidazolium group as compared to other acceptors previously employed. The transition strengths, even in B2TMC-2 $\left(\varepsilon=8156 \mathrm{M}^{-1} \mathrm{~cm}^{-1}\right)$, are markedly greater than those reported for TMC-2 $(\varepsilon=1840$ $\left.\mathrm{M}^{-1} \mathrm{~cm}^{-1}\right)$ and TMC-3 $\left(\varepsilon=2090 \mathrm{M}^{-1} \mathrm{~cm}^{-1}\right)$, likely reflecting the reduced torsional angles. The enhanced transition moment may also relate to the change in acceptor group, which dictates the attributes of the LUMO. Electronic structure calculations show that a large portion of the LUMO electron density is located on the bridging carbon, yielding a strong overlap with HOMO located on the donor fragment, particularly at intermediate torsional angles (Figure 12). These calculations also suggest that increased torsion largely manifests as localization of the LUMO on the benzimidazolium fragment, leading to larger $\Delta \mu_{\text {eg }}$ (Tables S7 and S8).

The BXTMC-2 CT transition in the 407-434 $\mathrm{nm}$ range is at much higher energy than that of previous twisted chromophores $\left(\lambda_{\mathrm{CT}}=569 \mathrm{~nm}\right.$ for TMC-2) due to the $\sim 0.5$ $\mathrm{eV}$ increase of the LUMO level as compared to TMC-2. This constitutes a significant advantage; a device utilizing BXTMC-

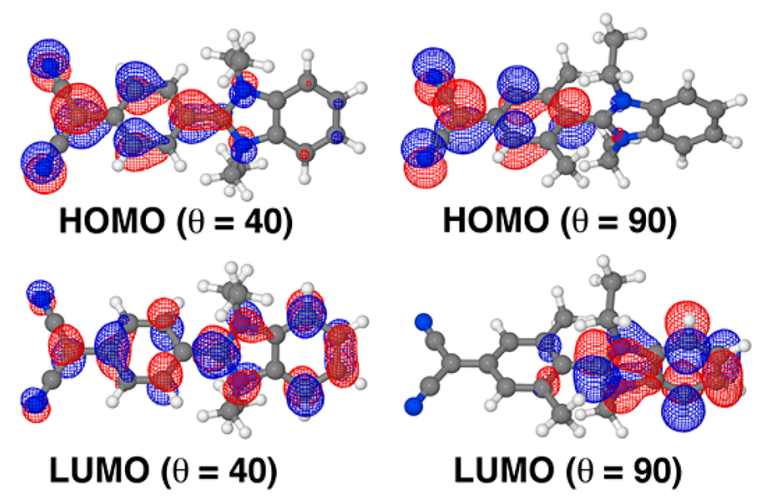

Figure 12. HOMO and LUMO contours of BXTMC-2 chromophores with $40^{\circ}$ and $90^{\circ}$ twist angles.
2 chromophores could operate without linear loss in a far broader spectral window. In fact, the absorption edge in polar solvents is close to the visible region cutoff, making a highperformance photonic device material with visible region transparency a distinct possibility.

Despite relatively large ground-state dipole moments, the BXTMC-2 chromophores are less sensitive to solvent environment than any previous second-order NLO TICT chromophore. The maximum measured solvatochromic shift (measured from $\mathrm{CHCl}_{3}$ to $\mathrm{MeOH}$ ) of B2TMC-2 is $-57 \mathrm{~nm}$ as compared to $\sim-180 \mathrm{~nm}$ for TMC-2, showing that replacement of the pyridinium fragment with benzimidazolium significantly reduces the dependence of the relative energy level spacing on the dielectric environment. Several factors may contribute to this observation: (1) the positioning of the bulky alkyl groups may reduce solvent interactions with the acceptor moiety, (2) the reduced CT distance versus TMC-2 decreases the dipole moment and therefore the importance of solvent stabilization, and (3) the benzimidazolium acceptor provides more effective stabilization of the ground-state positive charge than previous acceptor groups.

Monomeric NLO Response in DCM and DMF. The contribution of both $\mathrm{CH}_{2} / \mathrm{H}$ and $\mathrm{CH}_{2} / \mathrm{CH}_{3}$ steric interactions to the biaryl torsion allows access to intermediate solution twist angles that were not available in previous studies. The present evidence suggests that twist angle is directly related to $\mu \beta_{\text {vec }}$ and that the highest nonlinear response is achieved above $70^{\circ}$. The justification for large NLO response of this nature is now well-known to arise from the ZA/NQ balance in the molecule. ${ }^{19,50}$ Twisting, or otherwise disrupting conjugation, serves to simultaneously destabilize the ground state and create very large charge separation, thereby leading to giant dipole moments. The large NLO response is a consequence of these factors as shown by extensive experimental and computational work. This concept has also been demonstrated by recent theoretical work showing enhanced $\beta$ with disrupted conjugation as provided by large torsional angles. ${ }^{16,51}$

In the past, TICT chromophores performed poorly in polar environments despite marked decreases in aggregation., ${ }^{5,2}$ For TMC-2, NLO response was shown both experimentally and computationally to peak in moderately polar solvents and then diminish with increasing polarity. This behavior was attributed to the stabilization of the zwitterionic ground state relative to the excited-state manifold in highly polar environments. ${ }^{19,52,53}$ In the case of B2TMC-2, the similarity of NLO response observed in DMF and DCM is likely a result of reduced dependence of $E_{\mathrm{eg}}$ on solvent polarity as evidenced by relatively weak solvatochromic shifts $(\Delta \lambda$ in Table 2$)$. Of the TICT chromophores characterized to date, B2TMC-2 shows the highest reported nonlinearity in DMF at $10^{-3} \mathrm{M}$ (the highest measured concentration), highlighting the newfound ability to benefit from polar environments. It should be noted that, according to the two-level model, changes in the linear absorption should lead to decreased NLO response in DMF versus DCM. However, it was previously noted that the twolevel model does not always properly capture the impact of the dielectric medium. ${ }^{27}$

Concentration Dependence of NLO Response. In DCM, the aggregation tendencies of B2TMC-2 and TMC-2 are similar; high responses observed at low concentrations quickly diminish in the range of $10^{-4}-10^{-5} \mathrm{M}$. The observed aggregation tendencies are consistent with the tightly packed centrosymmetric unit cells in the crystal structures of B1TMC- 
2 and B2TMC-2. The relatively short linear alkyl groups used here do not prevent interactions between the donor group and the face of the benzimidazolium fragment as evidenced by the crystallographic packing. However, the design of BXTMC-2 allows for facile modification of the side chains; adding branched or dendritic groups has proven effective in the past and may be used to prevent close access to the face of the acceptor moiety. ${ }^{22,54}$ Aggregation of B2TMC-2 is less favorable in DMF as shown by the extension of high NLO response to concentrations in the range of $10^{-3}-10^{-4} \mathrm{M}$. Such improvements are encouraging but clearly must be supplemented by the aforementioned chemical modification of the side chains. Should this strategy prove effective in mitigating aggregation, it could be combined with the use of polar matrices, which had not been compatible with large NLO response in TICT chromophores until this point.

Intrinsic NLO Response. For systems of different size and properties to be compared, it is useful to examine the intrinsic hyperpolarizability defined by Kuzyk as $\beta_{\text {int }}=($ observed $\beta$ )/ (theoretical maximum, $\beta_{\max }$ ) for a structure with a particular number of polarizable electrons and a characteristic low-lying optical transition. ${ }^{1}$ Here, $\beta_{\max }$ is derived from linear absorbance measurements as described in section S2 of the Supporting Information. B2TMC-2 yields a remarkably high $\beta_{\text {int }}=0.37$, which is one of the largest values ever reported in the open literature (Table 4). Even modestly twisted BOTMC-2 exhibits

Table 4. Computed Theoretical $\left(\boldsymbol{\beta}_{\max }\right)$ and Intrinsic $\left(\boldsymbol{\beta}_{\text {int }}\right)$ and EFISH $\left(\beta_{\text {EFISH }}\right)$ Hyperpolarizability in DCM at $1907 \mathrm{~nm}$

\begin{tabular}{lccc} 
& \multicolumn{3}{c}{$\beta\left(\times 10^{-30}\right.$ esu $)$} \\
\cline { 2 - 4 } B0TMC-2 & $\beta_{\text {EFISH }}$ & $\beta_{\max }$ & $\beta_{\text {int }}$ \\
B1TMC-2 & -486 & -3370 & 0.14 \\
B2TMC-2 & -499 & -3070 & 0.16 \\
& -1056 & -2870 & 0.37 \\
\hline
\end{tabular}

a large $\beta_{\text {int }}=0.14$, still remarkably higher than the vast majority of efforts, which lie below $10^{-3 / 2}$. Thus, the BXTMC-2 chromophore series has proven capable of large NLO response arising from the efficient application of a small number of electrons.

\section{CONCLUSIONS}

A new series of twisted $\pi$-system electro-optic chromophores was synthesized by expeditious new routes and shown to be qualitatively similar to previous TICT generations with large ground-state dipole moments, low-lying CT transitions, and primarily zwitterionic/aromatic character. The same theories that justified NLO response in the past also apply here as evidenced by the dependence of $\mu \beta$ on twist angle. The introduction of steric hindrance using a benzimidazole moiety permits access to intermediate torsional angles and demonstrates the effect of experimentally twisting in this range for the first time.

The highlight of the present work is the strong NLO performance of chromophore B2TMC-2 in DMF, which represents a $5 \times$ increase over archetypical chromophore TMC2. We recognize that it is now possible to perform EFISH and E-O measurements in highly polar environments, opening new pathways to address detrimental aggregation. We are now pursuing promising directions involving modification of solvent polarity through addition of organic salts, means of artificially increasing polymer matrix polarity, and introduction of dendritic side chains. The use of highly polar matrices may be a key factor in preventing aggregate formation and realizing the exciting full potential of TICT chromophores.

\section{ASSOCIATED CONTENT}

\section{S Supporting Information}

The Supporting Information is available free of charge on the ACS Publications website at DOI: $10.1021 /$ jacs.8b04320.

X-ray crystallographic data for B0TMC-2 (CIF)

X-ray crystallographic data for B1TMC-2 (CIF)

X-ray crystallographic data for B2TMC-2 (CIF)

Detailed characterization of BXTMC-2 is available as well as crystallographic information and experimental procedures for the synthesis of all structures (PDF)

\section{AUTHOR INFORMATION}

\section{Corresponding Authors}

*t-marks@northwestern.edu

*elena.cariati@unimi.it

*alceo.macchioni@unipg.it

*cristiano.zuccaccia@unipg.it

ORCID

Stefania Righetto: 0000-0002-2537-3220

Cristiano Zuccaccia: 0000-0002-9835-2818

Elena Cariati: 0000-0003-1781-0360

Alceo Macchioni: 0000-0001-7866-8332

Tobin J. Marks: 0000-0001-8771-0141

Notes

The authors declare no competing financial interest.

\section{ACKNOWLEDGMENTS}

This work was supported by AFOSR MURI grant FA9550 14_1_0040 and made use of the IMSERC facility at Northwestern University, which has received support from the Soft and Hybrid Nanotechnology Experimental (SHyNE) Resource (NSF ECCS-1542205), the State of Illinois and International Institute for Nanotechnology (IIN). A. J.-T.L. thanks NDSEG for a Graduate Research Fellowship. A.M. and C.Z. thank PRIN $2015(20154 \times 9$ ATP 004) for financial support. The University of Perugia and MIUR are also acknowledged for financial support to the project AMIS through the program "Dipartimenti di Eccellenza-2018-2022". We also thank Dr. C. Malliakas for assistance in solving the crystal structure of BOTMC-2.

\section{REFERENCES}

(1) Kuzyk, M. G.; Singer, K. D.; Stegeman, G. I. Adv. Opt. Photonics 2013, 5, 4 .

(2) Benight, S. J.; Bale, D. H.; Olbricht, B. C.; Dalton, L. R. J. Mater. Chem. 2009, 19, 7466-7475.

(3) Sun, S.-S.; Dalton, L. R. Introduction to Organic Electronic and Optoelectronic Materials and Devices; Taylor \& Francis: New York, 2008.

(4) Cho, M. J.; Choi, D. H.; Sullivan, P. A.; Akelaitis, A. J. P.; Dalton, L. R. Prog. Polym. Sci. 2008, 33, 1013-1058.

(5) Kang, H.; Facchetti, A.; Jiang, H.; Cariati, E.; Righetto, S.; Ugo, R.; Zuccaccia, C.; Macchioni, A.; Stern, C. L.; Marks, T. J. J. Am. Chem. Soc. 2007, 129, 3267-3286.

(6) Kim, T.-D.; Luo, J.; Cheng, Y.-J.; Shi, Z.; Hau, S.; Jang, S.-H.; Zhou, X.-H.; Tian, Y.; Polishak, B.; Huang, S.; Ma, H.; Dalton, L.; Jen, A. K.-Y. J. Phys. Chem. C 2008, 112, 8091-9098. 
(7) Cheng, Y.-J.; Luo, J.; Huang, S.; Zhou, X.; Shi, Z.; Kim, T.-D.; Bale, D. H.; Takahashi, S.; Yick, A.; Steier, W.; Jen, A. K.-Y. Chem. Mater. 2008, 20, 5047-5054.

(8) Prasad, P. N.; Williams, D. J.: Introduction to Nonlinear Optical Effects in Molecules and Polymers; John Wiley \& Sons: New York, 1991.

(9) Meyers, F.; Marder, S. R.; Pierce, B. M.; Bredas, J. L. J. Am. Chem. Soc. 1994, 116, 10703-10714.

(10) Marder, S. R.; Kippelen, B.; Jen, A. K. Y.; Peyghambarian, N. Nature 1997, 388, 845.

(11) Gorman, C.; Marder, S. R. Chem. Mater. 1995, 7, 215-220.

(12) Zhang, C.; Dalton, L.; Oh, M.-C.; Zhang, H.; Steier, W. Chem. Mater. 2001, 13, 3043-3050.

(13) Galvan-Gonzalez, A.; Belfield, K. D.; Stegeman, G. I.; Canva, M.; Marder, S. R.; Staub, K.; Levina, G.; Twieg, R. J. J. Appl. Phys. 2003, 94, 756-763.

(14) Argouarch, G.; Veillard, R.; Roisnel, T.; Amar, A.; Boucekkine, A.; Singh, A.; Ledoux, I.; Paul, F. New J. Chem. 2011, 35, 2409.

(15) Kang, H.; Zhu, P.; Yang, Y.; Facchetti, A.; Marks, T. J. J. Am. Chem. Soc. 2004, 126, 15974-15975.

(16) Perez-Moreno, J.; Zhao, Y.; Clays, K.; Kuzyk, M. G. Opt. Lett. 2007, 32, 59-61.

(17) Leclercq, A.; Zojer, E.; Jang, S. H.; Barlow, S.; Geskin, V.; Jen, A. K.; Marder, S. R.; Bredas, J. L. J. Chem. Phys. 2006, 124, 044510.

(18) Albert, I. D. L.; Marks, T. J.; Ratner, M. A. J. Am. Chem. Soc. 1997, 119, 3155-3156.

(19) Brown, E. C.; Ratner, M. A.; Marks, T. J. J. Phys. Chem. B 2008, $112,44-50$.

(20) Kang, H.; Facchetti, A.; Stern, C. L.; Rheingold, W. S.; Marks, T. J. Org. Lett. 2005, 7, 3721.

(21) Shi, Y.; Frattarelli, D.; Watanabe, N.; Facchetti, A.; Cariati, E.; Righetto, S.; Tordin, E.; Zuccaccia, C.; Macchioni, A.; Wegener, S. L.; Stern, C. L.; Ratner, M. A.; Marks, T. J. J. Am. Chem. Soc. 2015, 137, 12521-12538.

(22) Wang, Y.; Frattarelli, D. L.; Facchetti, A.; Cariati, E.; Tordin, E.; Ugo, R.; Zuccaccia, C.; Macchioni, A.; Wegener, S. L.; Stern, C. L.; Ratner, M. A.; Marks, T. J. J. Phys. Chem. C 2008, 112, 8005-8015.

(23) Albert, I. D. L.; Marks, T. J.; Ratner, M. A. J. Am. Chem. Soc. 1998, 120, 11174-11181.

(24) Kanis, D. R.; Ratner, M. A.; Marks, T. J. Chem. Rev. 1994, 94, $195-242$.

(25) Tabor, C. E.; Dalton, L. R.; Kajzar, F.; Kaino, T.; Koike, Y. Proc. SPIE 2013, 8622, 86220J.

(26) Liao, Y.; Anderson, C. A.; Sullivan, P.; Akelaitis, A. J. P.; Robinson, B. H.; Dalton, L. Chem. Mater. 2006, 18, 1062-1067.

(27) Bale, D. H.; Eichinger, B. E.; Liang, W.; Li, X.; Dalton, L. R.; Robinson, B. H.; Reid, P. J. J. Phys. Chem. B 2011, 115, 3505-3513.

(28) Facelli, J. C.: Shielding Calculations: Perturbation Methods. In Encyclopedia of Nuclear Magnetic Resonance; John Wiley: London, 1996.

(29) Ramsey, N. F. Phys. Rev. 1950, 77, 567.

(30) Ramsey, N. F. Phys. Rev. 1950, 78, 699.

(31) Chen, B. C.; von Philipsborn, W.; Nagarajan, K. Helv. Chim. Acta 1983, 66, 1537-1555.

(32) Marelius, D. C.; Moore, C. E.; Rheingold, A. L.; Grotjahn, D. B. Beilstein J. Org. Chem. 2016, 12, 1334-1339.

(33) Nieto, C. I.; Cabildo, P.; García, M. A.; Claramunt, R. M.; Alkorta, I.; Elguero, J. Beilstein J. Org. Chem. 2014, 10, 1620-1629.

(34) Witanowski, M.; Stefaniak, L.; Webb, G. A.: Nitrogen NMR Spectroscopy. In Annual Reports on NMR Spectroscopy; Acedemic Press: London, 1993; Vol. 25.

(35) Mason, J.: Nitrogen NMR. In Encyclopedia of Nuclear Magnetic Resonance; John Wiley: London, 1996. pp 3222.

(36) Frohlich, R.; Musso, H. Chem. Ber. 1985, 118, 4649-4651.

(37) Allen, F. H.; Watson, D. G.; Brammer, L.; Orpen, A. G.; Taylor, R. International Tables for Crystallography 2006, C, 790-811.

(38) Lide, D. R. Tetrahedron 1962, 17, 125-134.

(39) Flandrois, P.; Chasseau, D. Acta Crystallogr., Sect. B: Struct. Crystallogr. Cryst. Chem. 1977, 33, 2744-2750.
(40) Zuccaccia, C.; Bellachioma, G.; Cardaci, G.; Macchioni, A. J. Am. Chem. Soc. 2001, 123, 11020-11028.

(41) Macchioni, A.; Magistrato, A.; Orabona, I.; Ruffo, F.; Rothlisberger, U.; Zuccaccia, C. New J. Chem. 2003, 27, 455-458.

(42) Neuhaus, D.; Williamson, M.: The Nuclear Overhauser Effect in Strucutral and Conformational Analysis; VCH Publishers: New York, 1989.

(43) Binev, Y. I.; Georgieva, M. K.; Novkova, S. I. Spectrochim. Acta, Part A 2003, 59, 3041-3052.

(44) McRae, E. G. J. Phys. Chem. 1957, 61, 562.

(45) Grabowski, Z. R.; Rotkiewicz, K.; Rettig, W. Chem. Rev. 2003, 103, 3899.

(46) Manohara, S. R.; Kumar, V. U.; Shivakumaraiah; Gerward, L. J. Mol. Liq. 2013, 181, 97.

(47) Sasaki, S.; Drummen, G. P. C.; Konishi, G.-i. J. Mater. Chem. C 2016, 4, 2731-2743.

(48) Kanis, D. R.; Lacroix, P. G.; Ratner, M. A.; Marks, T. J. J. Am. Chem. Soc. 1994, 116, 10089-10102.

(49) Isborn, C. M.; Davidson, E. R.; Robinson, B. H. J. Phys. Chem. A 2006, 110, 7189-7196.

(50) Teran, N. B.; He, G. S.; Baev, A.; Shi, Y.; Swihart, M. T.; Prasad, P. N.; Marks, T. J.; Reynolds, J. R. J. Am. Chem. Soc. 2016, 138, 6975-6984.

(51) Lytel, R.; Mossman, S. M.; Kuzyk, M. G. Opt. Lett. 2015, 40, 4735-4738.

(52) Abbotto, A.; Beverina, L.; Bradamante, S.; Facchetti, A.; Klein, C.; Pagani, G. A.; Redi-Abshiro, M.; Wortmann, R. Chem. - Eur. J. 2003, 9, 1991-2007.

(53) Chandra Ray, P. Chem. Phys. Lett. 2004, 395, 269-273.

(54) Hammond, S. R.; Sinness, J.; Dubbury, S.; Firestone, K. A.; Benedict, J. B.; Wawrzak, Z.; Clot, O.; Reid, P. J.; Dalton, L. R. J. Mater. Chem. 2012, 22, 6752. 